\title{
A NOTE ON INTERSECTIONS OF VALUATION IDEALS
}

\author{
CHARLES H. BRASE ${ }^{1}$
}

\begin{abstract}
In this note it is proved that if $R$ is an integral domain the set of valuation ideals of $R$ is closed under intersection if and only if the integral closure of $R$ is a valuation ring. Let $S$ be a domain which is integrally dependent on $R$ and contains the integral closure of $R$. Then the set of valuation ideals of $R$ is the same as the set of ideals of $R$ which contract from ideals of $S$ if and only if the set of valuation ideals of $R$ is closed under intersection.
\end{abstract}

In this paper all rings will be commutative rings with unity.

Definition. If $R$ is a domain, an ideal $I$ of $R$ is called a valuation ideal [6, p. 340] if $I$ is the contraction of an ideal of some valuation ring containing $R$ as a subring. If $I$ is any ideal of the domain $R$ we say the completion of $I$ is $I^{*}=\bigcap_{\alpha}\left(I V_{\alpha} \cap R\right)$ where $\left\{V_{\alpha}\right\}$ is the set of all valuation overrings of $R$. We say $I$ is complete if $I=I^{*}$.

Let $\mathscr{V}$ be the set of all valuation ideals of $R$. Let $\mathscr{S}$ be the set of all ideals of $R$ which can be written as an intersection of valuation ideals of $R$. Let $S$ be a domain containing $R$ as a subring. Let $C(S)$ be the set of all ideals of $R$ which are contracted from ideals of $S$.

If $S$ is integral over $R$, then it is well known [5, Theorem 3, p. 257] that the set of prime ideals of $R$ is contained in $C(S)$. Gilmer has proved [1, Theorem 2, p. 564] that $\mathscr{S} \subseteq C(S)$. Therefore when $S$ is integral over $R$ we have the containments $\mathscr{V} \subseteq \mathscr{S} \subseteq C(S)$. The following question seems to be rather natural. Assuming $S$ is integral over $R$, what are necessary and sufficient conditions that $\mathscr{V}=C(S)$ ?

Let $R$ be a domain. Let $\bar{R}$ denote the integral closure of $R$ in its quotient field. The following theorem answers the above question for the case in which $\bar{R} \subseteq S$.

The proof of this theorem uses the following result of Gilmer and Ohm [3, Lemma 2.3, p. 239]. If $I$ is a valuation ideal of a domain $R$ and $S, T$ are arbitrary subsets of $R$ then $S T \subseteq I$ implies $\left\{t^{2} \mid t \in T\right\} \subseteq I$ or $\left\{s^{2} \mid s \in S\right\} \subseteq I$.

Received by the editors May 1,1972 .

AMS (MOS) subject classifications (1970). Primary 13A15, 13B20; Secondary 13A05.

Key words and phrases. Valuation ideal, completion, integral closure.

${ }^{1}$ The author wishes to thank Professor Robert Gilmer for reading a first draft of this paper and for making suggestions which helped shorten the proof of the theorem.

(c) American Mathematical Society 1973 
THEOREM. Let $R$ be a domain. Let $S$ be a domain integral over $R$ such that $\bar{R} \subseteq S$. The following are equivalent:

(1) $\mathscr{V}=C(S)$.

(2) $\mathscr{V}=\mathscr{S}$.

(3) $\bar{R}$ is a valuation ring.

(4) The completion of each principal ideal of $R$ is a valuation ideal of $R$.

(5) An ideal of $R$ is a valuation ideal of $R$ if and only if it is complete.

Proof. The implications $(2) \Leftrightarrow(5),(1) \Rightarrow(2)$ and $(2) \Rightarrow(4)$ are clear.

$(2) \Rightarrow(3)$. Let $x$ and $y$ be nonzero elements of $R$. Since $\bar{R}$ is the intersection of all valuation rings which contain $R$ it follows by direct calculation that $x y \bar{R} \cap R=(x y) *$ is the intersection of the set of valuation ideals of $R$ containing $x y$. Therefore $x y \bar{R} \cap R$ is, by hypothesis, a valuation ideal. By the above-mentioned Lemma 2.3 of [3], $x^{2}$ or $y^{2}$ is in $x y \bar{R} \cap R$. Therefore $x$ is in $y \bar{R}$ or $y$ is in $x \bar{R}$. It follows that $\bar{R}$ is a valuation ring.

(3) $\Rightarrow(2)$. Let $I$ be a valuation ideal of $R$. Then $I V \cap R=I$ for some valuation overring $V$ of $R$. Hence $\bar{R} \subseteq V$ and $I \subseteq I \bar{R} \cap R \subseteq I V \cap R=I$. Therefore $I=I \bar{R} \cap R$. Now let $\left\{I_{\alpha}\right\}$ be any set of valuation ideals of $R$,

$$
\bigcap_{\alpha} I_{\alpha}=\bigcap_{\alpha}\left(I_{\alpha} \bar{R} \cap R\right)=\left(\bigcap_{\alpha} I_{\alpha} \bar{R}\right) \cap R .
$$

Since $\bar{R}$ is a valuation ring then $\bigcap_{\alpha} I_{\alpha}$ is a valuation ideal of $R$.

$(2) \Rightarrow(1)$. By the equivalence of (2) and (3) it follows that $\bar{R}$ is a valuation ring. If $I \in C(S)$ then

$$
I \subseteq I \bar{R} \cap R \subseteq I S \cap R=I .
$$

Therefore $I \in \mathscr{V}$. It follows that $C(S) \subseteq \mathscr{V}$ and consequently $C(S)=\mathscr{V}$.

$(4) \Rightarrow(3)$. In the proof of $(2) \Rightarrow(3)$ we only need the fact that $(a R)^{*}$ is a valuation ideal of $R$ for each $a$ in $R$.

REMARK 1. If $R$ is a Prüfer domain then, by [1, p. 288], $\mathscr{S}$ is the set of all ideals of $R$. Therefore if $R$ is a Prüfer domain and $S$ is any domain containing $R$ which is integral over $R$ then $\mathscr{S}=C(S)$.

REMARK 2. Let $R \subseteq S$ be domains such that $S$ is integral over $R$. Since $\mathscr{V} \subseteq \mathscr{S} \subseteq C(S)$ we see that $\mathscr{V}=C(S)$ implies $\mathscr{S}=C(S)$. However $\mathscr{S}=C(S)$ does not imply $\mathscr{V}=C(S)$. If $R$ is the ring of integers then $R=\bar{R}$. Let $S=\bar{R}$. Since $R$ is Prüfer, $\mathscr{S}=C(S)$. In a Prüfer ring $\mathscr{S}$ is the set of all ideals of $R$. But the set of valuation ideals of the ring of integers is just the set of primary ideals (see [3]). Therefore $\mathscr{V} \neq C(S)$.

REMARK 3. If the set of valuation ideals of a domain $R$ is closed under intersection then it is closed under addition. But the converse is not true. For if $\mathscr{S}=\mathscr{V}$ then $\bar{R}$ is a valuation ring and all valuation ideals of $R$ contract from $\bar{R}$. Therefore the valuation ideals of $R$ are linearly ordered under 
containment. The valuation ideals of the integers are closed under addition but not under intersection.

REMARK 4. The method of proof of the Theorem can be used to show that the valuation ideals of a domain $R$ are closed under finite intersection if and only if every finite intersection of valuation overrings of $R$ is a valuation ring.

\section{REFERENCES}

1. R. W. Gilmer, Jr., Contracted ideals with respect to integral extensions, Duke Math. J. 34 (1967), 561-571. MR 36 \#1428.

2. - Multiplicative ideal theory, Queen's Papers in Pure and Appl. Math., no. 12, Queen's University, Kingston, Ont., 1968. MR 37 \#5198.

3. R. Gilmer and J. Ohm, Primary ideals and valuation ideals, Trans. Amer. Math. Soc. 117 (1965), 237-250. MR 30 \#114.

4. M. Larsen and P. McCarthy, Multiplicative theory of ideals, Academic Press, New York, 1971.

5. O. Zariski and P. Samuel, Commutative algebra. Vol. 1, University Ser. in Higher Math., Van Nostrand, Princeton, N.J., 1958. MR 19, 833.

6. - Commutative algebra. Vol. 2, University Ser. in Higher Math., Van Nostrand, Princeton, N.J., 1960. MR 22 \#11006.

Department of Mathematics, University of Hawail, Honolulu, Hawail 96822 